\title{
The Association between KIF6 Single Nucleotide Polymorphism rs20455 and Serum Lipids in Filipino-American Women
}

\author{
Irma B. Ancheta, ${ }^{1}$ Cynthia A. Battie, ${ }^{1}$ Dan Richard, ${ }^{1}$ Christine V. Ancheta, ${ }^{2}$ \\ Nancy Borja-Hart, ${ }^{3}$ Annabelle S. Volgman, ${ }^{4}$ and Yvette Conley ${ }^{5}$ \\ ${ }^{1}$ School of Nursing, University of North Florida, 1 UNF Drive, Jacksonville, FL 32224, USA \\ ${ }^{2}$ University of South Florida, Tampa, FL 33612, USA \\ ${ }^{3}$ East Coast Institute for Research, Jacksonville, FL 32223, USA \\ ${ }^{4}$ Rush University Medical Center, Chicago, IL 60612, USA \\ ${ }^{5}$ University Pittsburgh, Pittsburgh, PA 15213, USA
}

Correspondence should be addressed to Irma B. Ancheta; i.ancheta@unf.edu

Received 22 June 2013; Revised 21 September 2013; Accepted 21 October 2013; Published 23 January 2014

Academic Editor: Adebowale A. Adeyemo

Copyright (C) 2014 Irma B. Ancheta et al. This is an open access article distributed under the Creative Commons Attribution License, which permits unrestricted use, distribution, and reproduction in any medium, provided the original work is properly cited.

\begin{abstract}
The Trp719Arg allele of KIF6 rs20455, a putative risk factor for CHD especially in those with elevated low-density lipoprotein cholesterol (LDL-C), was investigated in Filipino-American women (FAW, $n=235$ ) participating in health screenings in four cities. The rs20455 genotype of each subject was determined by a multiplex assay using a Luminex-OLA procedure. The risk allele Trp719Arg was present in $77 \%$ of the subjects. The genotype distribution was $23 \% \operatorname{Trp} / \operatorname{Trp}, 51 \% \operatorname{Arg} / \operatorname{Trp}$, and $26 \%$ Arg/Arg. Genotype did not predict the presence of CHD risk factors. Moreover, LDL-C, HDL-C, and triglycerides mean values did not vary as a function of genotype. However, those with the Arg/Arg genotype on statin medication exhibited a significantly higher mean triglycerides level $(P<0.01)$. Approximately $60 \%$ of participants regardless of genotype exhibited LDL-C levels $\geq 100 \mathrm{mg} / \mathrm{dL}$ but were not taking medication. Approximately 43\% of those with the Trp719Arg risk allele on statins exhibited elevated LDL-C levels. Our study suggests that the Trp719Arg allele of KIF 6 rs20455 is common among Filipino-American women; thus, even with borderline LDL-C levels would benefit from statin treatment. Secondly, many participants did not exhibit guideline recommended LDL-C levels including many who were on statin drugs.
\end{abstract}

\section{Introduction}

Coronary heart disease is a multifactorial and complex disease resulting from the interaction of genes and environmental factors [1-5]. Genetics plays an important role in determining the inherent CHD vulnerability and in determining how a person responds to statin therapy. KIF6 is a member of the kinesin family, a class of motor proteins that are involved in the intracellular transport of membrane organelles, messenger RNA's and other protein complexes along microtubules [6-10]. Several studies focusing on Caucasians have reported that the Trp719Arg allele of single nucleotide polymorphism (SNP) rs20455 in the KIF6 gene is associated with CHD and that carriers who carry one or two copies of the risk allele respond better to statin therapy than noncarriers [10-15]. The prevalence among Caucasians is about $59 \%$ and the risk ratio has been stated to be 1.22 (95\% confidence intervals 1.12-1.32) [15]. This association with increased risk was present in African Americans, who have very high numbers $(\sim 90 \%)$ with the risk allele [15]. Chinese and Japanese also have been reported to have a high frequency $(\sim 70 \%)$ of the risk allele [15]. Other investigators were unable to detect the association of the rs20455 allele with increased CHD risk [16] even with a large sample size [17]. Ference et al. [18] carried out a large meta-analysis with 88,535 subjects in 8 randomized statin trials examining the role of KIF6 Trp719Arg allele in CHD and concluded that the risk allele increases the vulnerability to elevated LDL-C cholesterol. Thus, the KIF6 genetic variant may be a predictor 
of CHD in those who have elevated LDL-C. Indeed, carriers of the Trp719Argallele may have a greater increase in CHD risk per unit increase in LDL and a greater reduction in CHD risk per unit decrease in LDL, compared to noncarriers [18]. Indeed, the number needed to treat with statins to prevent a single CHD event ranged from 10 to 20 for the Trp719Arg carriers compared to $>80$ for noncarriers in a large meta-analysis [15]. Furthermore, the results of Ference et al. [18] suggest that the differential vulnerability to LDL$\mathrm{C}$ based upon genotype may explain the reason for the prior disagreement in studies.

To the best of our knowledge despite a plethora of evidence, no studies of KIF6 Trp719Arg allele have been conducted in the Filipino population. Filipinos are the second largest minority population of Asians in America [19] and have the highest prevalence of hypertension [20], type 2 diabetes [21, 22], and metabolic syndrome [23] compared with other Asian subgroups. The heterogeneity of CHD risk factors among Asians is well established [24] and the allelic frequency distribution of rs20455 has been shown to vary across populations [15]. Scientific gaps still exist regarding the role of genetics in the predisposition to $\mathrm{CHD}$ in various populations. Since the rs20455 variant has not been studied in those of Filipino descent, we investigated its prevalence in association with plasma lipid levels in a cohort of Filipino American women, who attended community-based health screenings at various locations throughout the USA.

\section{Materials and Methods}

2.1. Study Design, Population, and Recruitment Procedure. Approval of the University Institutional Research Board (IRB) was obtained prior to the conduct of this study. This study is a descriptive, cross-sectional study of Filipino American women $(n=234)$, who participated in a cardiovascular health screening at their places of worship or cultural centers during 2011-2013. Subjects were between 40 and 65 years of age. Health screenings were performed in various US cities: Jacksonville, FL; Chicago, IL; Tampa, FL, and San Francisco, CA. In order to recruit a wide variety of subjects, advertisements were posted in church bulletins and at community organizations and stores frequently visited by Filipino women. These flyers specified inclusion and exclusion criteria and the need to be fasting for at least 12 hours on the day of the study. Participants were instructed to bring all prescription and nonprescription medications to the study site.

Participants were enrolled if they were women, selfidentified as Filipino by ethnicity, agreed to volunteer for the study, and were fasting for 12 hours. Women who had severe arthritis, any autoimmune disorder, and a recent cancer diagnosis and/or presented with any infection or severe inflammation were excluded from the study important for the measurement of inflammatory markers. Informed consent was obtained after the study purpose was carefully explained. Prior to giving consent, participants were given ample time to ask questions or state concerns regarding the study. Total time for participation in the study was approximately $45 \mathrm{~min}$ per participant.

2.2. Screening Protocol. A demographic and clinical information questionnaire, including participants' medical history and current medications, was completed for each subject by trained research staff. The clinical information questionnaire included family history, smoking history, participant's medical history, and current medications. Upon completion of the survey, blood pressure was obtained using a standard Omron digital HEM-705CP sphygmomanometer on the nondominant arm, after the participant had been seated for $10 \mathrm{~min}$. Measurements were repeated three times with $5 \mathrm{~min}$ in between each reading. Subsequently, weight and height were measured using a standard Tanita weighing scale (WB3000). Waist circumference was measured using a tension tape guided by the NHANES measurement protocol. Lastly, a licensed phlebotomist drew $5 \mathrm{~mL}$ of blood via venipuncture from each participant for a series of tests including the standard lipid panel, hemoglobin A1C, serum glucose, a cardiovascular inflammatory biomarker (high-sensitivity Creactive protein or hs-CRP), and genetic assays. The blood was captured in three test tubes containing EDTA. Sample tubes were labeled with numbers only, centrifuged, and immediately frozen. At the end of each screening session, frozen samples were sent to a CLIA certified laboratory where assays were performed using standard clinical protocols. The lipid panel assay included total cholesterol, triglycerides, high-density lipoprotein (HDL), and low-density lipoprotein (LDL-C) (Roche Modular methodology) performed at Berkeley Heartlab, Inc. (Alameda, CA). Plasma hs-CRP concentration was determined using an automated immunoturbidimetric assay (Roche Modular methodology, Berkeley Heartlab Inc.).

2.3. Cardiovascular Risk Factors. The operational definition for CHD risk factors for the study was based on the following guidelines. For blood pressure values, the American Heart Association and the Joint National Committee for the Prevention, Detection, Evaluation, and Treatment of High Blood Pressure (JNC 7) guidelines for the normal range of blood pressure were used $(\geq 120 \mathrm{mmHg}$ systolic and/or diastolic blood pressure of $\leq 80 \mathrm{mmHg}$ ). We used the criteria of the World Health Organization regarding cut-off for the body mass index (BMI). The National Cholesterol Education Program (NCEP) Adult Treatment Panel III guidelines were used to define the cholesterol normal reference values, namely, total cholesterol (TC) $\geq 200 \mathrm{mg} / \mathrm{dL}$, triglycerides (TGL) $\geq 150 \mathrm{mg} / \mathrm{dL}$, high-density lipoproteins cholesterol (HDL-C) $\leq 50 \mathrm{mg} / \mathrm{dL}$, low-density lipoprotein cholesterol (LDL-C) $\geq 100 \mathrm{mg} / \mathrm{dL}$, fasting blood glucose $(\mathrm{FBG}) \geq 100 \mathrm{mg} / \mathrm{dL}(5.6 \mathrm{mmol} / \mathrm{L})$, and waist circumference $\geq 35$ inches $(88 \mathrm{~cm})$. The American Diabetes Association criteria for diabetes were used: hemoglobin A1C $\geq 6.5 \%$ and fasting plasma glucose $\geq 126 \mathrm{mg} / \mathrm{dL}$. Metabolic syndrome was defined using both the International Diabetes federation and the National Cholesterol Education ProgramAdult Treatment Panel III criteria. 
TABLE 1: Demographic characteristics of the Filipino-American women subjects.

\begin{tabular}{|c|c|c|}
\hline Demographic characteristics & $\begin{array}{c}\text { Mean } \pm \text { SD } \\
(n=234)\end{array}$ & Frequency $(\%)$ \\
\hline Age & $51.5 \pm 7$ & - \\
\hline No. of years in the USA & $24.0 \pm 13$ & - \\
\hline Age-arrival in USA & $31.0 \pm 11$ & - \\
\hline \multicolumn{3}{|l|}{ Marital status } \\
\hline Single & & 8 \\
\hline Married & & 73 \\
\hline Divorced/widow & & 19 \\
\hline Philippine-born & & 98 \\
\hline \multicolumn{3}{|l|}{ Residency in the USA } \\
\hline$<5$ years & & 11 \\
\hline $5-10$ years & & 6 \\
\hline $10-20$ years & & 26 \\
\hline Over 20 years & & 57 \\
\hline \multicolumn{3}{|l|}{ Income } \\
\hline$<\$ 12,000 /$ year & & 17 \\
\hline$\$ 13,000-\$ 40,000$ & & 47 \\
\hline$\$ 41,000-\$ 69,000$ & & 20 \\
\hline$\$ 70,000$ and above & & 16 \\
\hline \multicolumn{3}{|l|}{ Education } \\
\hline High school & & 18 \\
\hline Some college & & 21 \\
\hline 4 year degree & & 52 \\
\hline Graduate degree & & 9 \\
\hline \multicolumn{3}{|l|}{ Occupation } \\
\hline Health occupations & & 34 \\
\hline \multicolumn{3}{|l|}{ Healthcare insurance } \\
\hline With insurance & & 87 \\
\hline
\end{tabular}

2.4. Genetic Analysis. Celera research reagents were used to genotype the rs20455 SNP of each subject in a singletube assay using a Luminex-OLA procedure as described previously [25]. The procedure included amplification of genomic DNA ( $\sim 3 \mathrm{ng})$ by multiplex PCR followed by multiplex OLA. The resulting ligation products were hybridized to Luminex xMAP microspheres and labeled by the reporter molecule SA-PE. The xMAP microspheres were analyzed on a Luminex 100 or a Luminex 200 systems. Genotypes were subsequently determined using the Celera allele calling software as described in Iannone et al. [25].

2.5. Data Analysis. A confidential, password-secured database was used for data entry, management, and analysis. Inconsistencies were checked, and the data descriptions were verified by the principal investigator and the statistician. Data were analyzed using the Statistical Package for the Social Sciences (SPSS, version 19) and GraphPad Prism 5 software. Means \pm standard deviations were determined for all continuous variables and number and percentage
TABLE 2: Morphometric measurements and cardiovascular risk factors of the Filipino-American women participants $(n=235)$.

\begin{tabular}{lcc}
\hline Measurement & Means \pm SD & $\begin{array}{c}\text { Percent of group } \\
\text { (risk level) }\end{array}$ \\
\hline Weight (lbs.) & $151 \pm 22$ & \\
Height (inches) & $61 \pm 2$ & $37\left(\geq 25 \mathrm{~kg} / \mathrm{m}^{2}\right)$ \\
Body mass index & $29 \pm 4$ & $15\left(\geq 30 \mathrm{~kg} / \mathrm{m}^{2}\right)$ \\
Waist circumference & $40 \pm 4$ & $79(\geq 35 \mathrm{inches})$ \\
Systolic blood pressure & $131 \pm 19$ & $64(\geq 120 \mathrm{mmHg})$ \\
Diastolic blood pressure & $87 \pm 10$ & $61(\leq 80 \mathrm{mmHg})$ \\
Fasting blood glucose & $101 \pm 25$ & $38(\geq 100 \mathrm{mg} / \mathrm{dL})$ \\
Hemoglobin A1C & $6.0 \pm 0.8$ & $36(\geq 6.5 \%)$ \\
Total cholesterol & $201 \pm 45$ & $44(\geq 200 \mathrm{mg} / \mathrm{dL})$ \\
Triglycerides & $116 \pm 67$ & $19(\geq 150 \mathrm{mg} / \mathrm{dL})$ \\
Low-density lipoprotein-C & $122 \pm 35$ & $61(\geq 100 \mathrm{mg} / \mathrm{dl})$ \\
High-density lipoprotein-C & $62 \pm 15$ & $21(\leq 50 \mathrm{mg} / \mathrm{dL})$ \\
High-Sensitivity & $1.97 \pm 3.0$ & $13(<3.0 \mathrm{mg} / \mathrm{L})$ \\
C-Reactive protein & & 56 \\
Metabolic syndrome & & 3 \\
Smoking & & 48 \\
Family history & & 5
\end{tabular}

Note: metabolic syndrome was defined by both the International Diabetes Federation (IDF) and the National Cholesterol Education Program-Adult Treatment Panel III (NCEP/ATP III) criteria.

were determined for categorical variables. Analysis of variance (ANOVA) was used to compare group means and multiple stepwise linear regression determined whether risk alleles significantly predicted CHD risk factors, controlling for alternative predictors of $\mathrm{CHD}$ risks. Dichotomous variables were analyzed using Fisher's chi-square tests for independence with Yates continuity correction. Statistical significance was set at $P<0.05$.

\section{Results}

3.1. Demographic Characteristics. Demographic characteristics of the nonrelated Filipino-American women $(n=234)$ enrolled in the study are shown in Table 1. The mean age of the women was $55.4 \pm 7.1$ years old and the majority (98\%) were born in the Philippines with the mean length of residency in USA of $24.1 \pm 13.1$ years. The mean age upon arrival in the USA was $31.1 \pm 10.9$ years. The majority (75\%) were married and many had completed a college degree and were employed in health-related professions. Most of the participants (88\%) had some sort of insurance including private insurance (49\%).

3.2. Cardiovascular Risk Factors. Clinical and morphometric measurements are shown in Table 2. Participants had a mean height of $5^{\prime} 1^{\prime \prime}$ and mean weight of $151 \mathrm{lbs}$. and $50 \%$ of participants were considered either overweight or obese as indicated by BMI. The majority were classified as prehypertensive because blood pressure was over the criterion 
TABLE 3: Selective cardiovascular disease risk factors as a function of the rs20455 genotype and statin medication usage.

\begin{tabular}{|c|c|c|c|c|}
\hline Risk factors & $\begin{array}{c}\text { AA } \\
\text { Trp/Trp } \\
n=53 \\
23 \%\end{array}$ & $\begin{array}{c}\text { AG } \\
\text { Arg/Trp } \\
n=120 \\
51 \%\end{array}$ & $\begin{array}{c}\text { GG } \\
\text { Arg/Arg } \\
n=62 \\
26 \%\end{array}$ & $\begin{array}{l}\text { ANOVA } \\
P \text { value }\end{array}$ \\
\hline \multicolumn{5}{|l|}{ Age } \\
\hline Total & $52 \pm 7$ & $54 \pm 7$ & $52 \pm 6.9$ & 0.09 \\
\hline No statins & $51 \pm 10$ & $52 \pm 9$ & $50 \pm 10$ & 0.28 \\
\hline Statins & $52 \pm 4$ & $54 \pm 6$ & $52 \pm 7$ & \\
\hline \multicolumn{5}{|l|}{ HDL-C } \\
\hline Total & $60 \pm 14$ & $64 \pm 15$ & $61 \pm 16$ & 0.21 \\
\hline No statins & $60 \pm 16$ & $65 \pm 7$ & $63 \pm 15$ & 0.06 \\
\hline Statins & $56 \pm 11$ & $61 \pm 15$ & $52 \pm 14$ & \\
\hline \multicolumn{5}{|l|}{ LDL-C } \\
\hline Total & $114 \pm 36$ & $113 \pm 38$ & $113 \pm 37$ & 0.71 \\
\hline No statins & $118 \pm 39$ & $119 \pm 39$ & $116 \pm 34$ & 0.59 \\
\hline Statins & $106 \pm 32$ & $101 \pm 36$ & $113 \pm 40$ & \\
\hline \multicolumn{5}{|l|}{ TG } \\
\hline Total & $119 \pm 58$ & $109 \pm 67$ & $130 \pm 74^{1,2}$ & 0.009 \\
\hline No statins & $118 \pm 60$ & $107 \pm 74$ & $111 \pm 54$ & \\
\hline Statins & $121 \pm 50$ & $113 \pm 39$ & $177 \pm 97^{3}$ & 0.002 \\
\hline \multicolumn{5}{|l|}{ Waist Circ } \\
\hline Total & $37 \pm 7$ & $37 \pm 6$ & $36 \pm 7$ & \\
\hline No statins & $36 \pm 6$ & $37 \pm 4$ & $37 \pm 4$ & 0.93 \\
\hline Statins & $37 \pm 4$ & $36 \pm 7$ & $37 \pm 3$ & \\
\hline \multicolumn{5}{|l|}{ BMI } \\
\hline Total & $26 \pm 4$ & $28 \pm 4$ & $26 \pm 4$ & 0.06 \\
\hline No statins & $26 \pm 4$ & $27 \pm 4$ & $25 \pm 5$ & \\
\hline Statins & $30 \pm 5$ & $30 \pm 4$ & $27 \pm 5$ & 0.15 \\
\hline \multicolumn{5}{|l|}{ Hs-CRP } \\
\hline Total & $1.41 \pm 1.5$ & $1.86 \pm 2.2$ & $1.55 \pm 2.1$ & 0.45 \\
\hline No statins & $2.1 \pm 3.9$ & $2.2 \pm 3.3$ & $1.6 \pm 1.7$ & \\
\hline Statins & $1.6 \pm 2.9$ & $1.4 \pm 1.9$ & $2.2 \pm 3.0$ & 0.87 \\
\hline \multicolumn{5}{|l|}{ HbAlc } \\
\hline Total & $5.8 \pm 0.46$ & $5.8 \pm 0.49$ & $6.01 \pm 0.97$ & 0.39 \\
\hline No statins & $5.8 \pm 0.48$ & $5.8 \pm 0.48$ & $5.9 \pm 0.66$ & \\
\hline Statins & $6.3 \pm 1.2$ & $6.1 \pm 0.54$ & $6.4 \pm 1.5$ & 0.17 \\
\hline
\end{tabular}

Note: HDL-C: high-density lipoprotein cholesterol; LDL-C: low-density lipoprotein-cholesterol; TG: triglycerides; Waist Circ: waist circumference; BMI: body mass index; hs-CRP: high-sensitivity C-reactive protein; HbAlc: Hemoglobin Alc. ${ }^{1} P<0.05 \operatorname{Trp} / \operatorname{Trp}$ versus Arg/Arg, ${ }^{2} P<0.01 \mathrm{Arg} / \mathrm{Trp}$ versus $\mathrm{Arg} / \mathrm{Arg}$, and ${ }^{3} \mathrm{Arg} / \mathrm{Arg}$ versus all other groups $P<0.001$; AA no statins $n=22$, statins $n=12$; AG no statins $n=48$, statins $n=21$; GG no statins $n=26$, statins $n=16$

set forth by the JNC 7. Approximately $36 \%$ of the women had diabetes as indicated by the levels of hemoglobin AlC. A high percentage $(61 \%)$ of the participants had elevated LDL-C but the percent of the group (21\%) with unhealthy HDL and elevated triglycerides (19\%) was much smaller. Elevated hs-CRP was seen in 13\% of the group. Approximately $50 \%$ of participants were classified as having metabolic
TABLE 4: Multiple regression analysis of cardiovascular risk factors.

\begin{tabular}{lccc}
\hline Variables & $\begin{array}{c}\text { TG T ratio } \\
(P \text { value })\end{array}$ & $\begin{array}{c}\text { LDL T ratio } \\
(P \text { value })\end{array}$ & $\begin{array}{c}\text { HDL T ratio } \\
(P \text { value })\end{array}$ \\
\hline Genotype & $0.57(0.27)$ & $0.18(0.86)$ & $1.11(0.24)$ \\
HbAlc & $3.42(0.0008)$ & $0.75(0.45)$ & $3.36(0.001)$ \\
hs-CRP & $0.97(0.34)$ & $0.41(0.68)$ & $1.92(0.06)$ \\
Age & $0.46(0.64)$ & $2.32(0.02)$ & $1.15(0.25)$ \\
BMI & $0.56(0.57)$ & $1.23(0.22)$ & $0.22(0.86)$ \\
\hline
\end{tabular}

Note: HbAlc: hemoglobin Alc; hs-CRP: high-sensitivity C-reactive protein.

syndrome. About $50 \%$ of participants self-reported a family history of heart disease, indicated by stroke and/or CHD events of parents and immediate siblings. Only $3 \%$ of the participants smoked and thus smoking was not thought to be a confounder in this study.

3.3. KIF6 rs20455 Genotype and CHD Risk Factors. The distribution of genotypes is as follows: $23 \%$ AA (Trp/Trp), 51\% AG (Arg/Trp), and 26\% GG (Arg/Arg). Thus, a majority (77\%) of participants have at least one copy of the risk allele. This SNP was found to be in Hardy Weinberg equilibrium. There were no differences in mean age, BMI, waist circumference, hs-CRP, or HbAlc between the groups (Table 3).

Differences in lipid risk factors as a function of the rs20455 genotype and statin use are shown in Table 3. The mean value of TG was significantly elevated in the GG genotype $(\mathrm{Arg} / \mathrm{Arg})$ group $(P<0.009)$ but further analysis indicated that this elevation was only seen in those of this genotype taking statin medication. Multiple regression analyses confirmed a lack of association of genotype with TG levels but indicated a strong association with diabetes $(P=0.0008)$ indicated by HbAlc (Table 4$)$. The prevalence of high TG $(\geq 150 \mathrm{mg} / \mathrm{dL})$ as a function of genotype was $10 \%$ of AA (Trp/Trp), $16 \%$ of AG (Trp/Arg), and 38\% of GG (Arg/Arg) genotype (Fisher's exact $P<0.001$ ) indicating that those subjects with the GG (Arg/Arg) genotype were more likely to have elevated TG levels. Similar to TG, there was a lack of association of genotype with HDL-C levels but an association with diabetes $(P=0.001)$. No differences in mean values of HDL-C between groups were apparent. No association of LDL-C with genotype nor differences in mean values were seen as a function of genotype or statin usage but an association of LDL-C with age was seen $(P=0.02)$.

We also investigated the prevalence of elevated LDL-C as a function of genotype and statin use. Many participants (60$65 \%$; Table 5) had elevated LDL-C and were not being treated with statins including $65 \%$ of those with the Trp719Arg allele. Moreover, many in each genotype who were on statin medication exhibited elevated LDL-C including $43 \%$ of those with the Trp719Arg allele.

\section{Discussion}

The major finding of this study is a high prevalence (0.70) of the Trp719Arg allele in the Filipino-American women participants. Moreover, a high percent of women with the risk 
TABle 5: Percent of participants with elevated LDL as a function of genotype and statin usage.

\begin{tabular}{lccccc}
\hline Medications & $\begin{array}{c}\text { Trp/Trp } \\
\text { (AA) }\end{array}$ & $\begin{array}{c}\text { Arg/Trp } \\
\text { (AG) }\end{array}$ & $\begin{array}{c}\text { Arg/Arg } \\
\text { (GG) }\end{array}$ & $\begin{array}{c}\text { Arg/Trp } \\
\text { Arg/Arg } \\
\text { (AG + GG) }\end{array}$ & $\begin{array}{c}\text { Fisher's exact test } \\
P \text { Value }\end{array}$ \\
\hline No statins & $n=22$ & $n=48$ & $n=26$ & $n=74$ & \\
\% elevated LDL-C & $69 \%$ & $67 \%$ & $62 \%$ & $65 \%$ & 0.41 \\
Statins & $n=12$ & $n=21$ & $n=16$ & $n=37$ & \\
\% elevated LDL-C & $58 \%$ & $33 \%$ & $56 \%$ & $43 \%$ & 0.31 \\
\hline
\end{tabular}

allele and elevated LDL-C levels were not being treated with statin medications and a significant number of those with the risk allele on statin medications still exhibited elevated LDL-C levels. Results from the current study are novel since an extensive search in the literature revealed no studies describing the prevalence of rs20455 SNP of KIF6 and its association with lipid levels in Filipino-American women. Our study may be the first in which the CVD rs20455 risk allele and its association to LDL-C were determined in this group of understudied minority women. Our current results showed that the majority of the FAW participants carried a single (heterozygote) or double (homozygote) copy of the risk allele. The carrier frequency seen for Filipino-American women is similar to those reported by Li et al. [15] for Asians (Japanese and Chinese) which was $72 \%$ based upon HapMap and Celera data. Thus, the carrier frequency may be higher in Asians than Caucasians who had a prevalence of $59 \%$ in one study [15]. Peng et al. [9] have reported differences in the rs20455 allele frequencies across populations.

Another finding of our study is that TG levels were significantly elevated in individuals with the Arg/Arg genotype and on statin medication. This result could be interpreted as those with Arg/Arg genotype have higher TG levels when they have elevated LDL-C but, given the small subject number, further study is warranted. Wu et al. [10] in a case-control study evaluated the association of KIF6 rs20455 SNP with angiographic CAD and serum lipid levels in the Han Chinese population of northern China. Their results demonstrated no significant differences in genotype and allele frequency between the cases (angiographic CAD) and controls $(P>$ 0.05 ). However, further analysis showed that nonfatal MI risk and TG levels were significantly higher in 719Arg carriers compared to noncarriers $(P<0.05)[10]$.

Our findings demonstrated that there were no differences in HDL or LDL-C levels as a function of genotype; however, this finding must be viewed with caution because the sample size may not be sufficiently powered to discern an association of genotype with lipid levels. Importantly, there were a significant number of Filipino-American women, including many with the Trp719Arg allele, who had elevated LDL-C but were not being treated with statins. Others were being treated but had not achieved recommended levels of LDL-C. Although studies regarding KIF6's polymorphism and risk for CHD are equivocal, the KIF6 genetic variant may be a predictor of CHD in those who have elevated LDL-C as described in the Introduction [10-18]. Given that the number needed to treat with statins to prevent a single $\mathrm{CHD}$ event ranged from 10 to 20 for the Trp719Arg carriers, it is imperative that those with this allele be treated for elevated LDL-C [15]. The elevated LDL-C in participants on statin medication could indicate noncompliance with statin medications due to muscle pains or other side effects of the drugs; however, we did not capture data about nonadherence to prescribed cholesterollowering medication [26, 27]. Additionally, elevated LDL$\mathrm{C}$ levels despite use of statins in this population may be conceivably due to genetic differences of this population [9]. These results may also be due to the differential performance of lipophilic and hydrophilic statins [28, 29]. Bonsu et al. [28] conducted a systematic review and meta-analysis of lipophilic and hydrophilic statins on heart failure patients. The investigators claimed that there are differences in the pleotropic effects of statin medications due to differences in lipophilicity in the type of statin medications. Lipophilic statins utilize passive diffusion for entering cells while hydrophilic statins use carrier-mediated process for uptake in the liver cells. Although there are controversial outcomes on the effects of lipophilic statins, this group of statins appear to show more beneficial effects than those of hydrophilic statins, though improved outcomes have been reported for rosuvastatin in the Controlled Rosuvastatin in Multinational Trial in Heart Failure (CORONA) and the Gruppo Italiano per lo Studio della Sopravvivenza nell'Infarto Miocardico Heart Failure (GISSI-HF) trials [29]. Regardless of cause, further research is required to determine why LDL-C is not at optimal levels among Filipino-American women even when taking cholesterol-lowering medications. Our study indicates that this population needs to be more carefully monitored and treated for elevated LDL-C. Specific statin medications may have adverse effects in Asian populations [30]. In 2005, the US Food and Drug Administration provided additional safety label warning for rosuvastatin since a pharmacokinetic study that included a diverse population of Asians showed that rosuvastatin drug levels were found to be approximately twofold higher when compared with the Caucasian control group. This in effect may produce increased risk of muscle myopathies and risk of kidney failure in Asians [30].

Many clinical trials have shown the benefit of lowering cholesterol levels even in those with relatively normal cholesterol levels and no previous myocardial infarctions [31-33]. One clinical trial of participants with myocardial infarction and plasma total cholesterol $<240 \mathrm{mg} / \mathrm{dL}$ and LDLC of $115-174 \mathrm{mg} / \mathrm{dL}$ randomized to $40 \mathrm{mg}$ of pravastatin 
per day or placebo had $10.2 \%$ events in the pravastatin group and $13 \%$ events in the placebo group indicating that cholesterol-lowering treatment is beneficial for CAD patients with borderline cholesterol levels [31]. Pravastatin lowered cholesterol by $20 \%$ and LDL-C by $26 \%$ and reduced MI incidence in men with hypercholesterolemia and no previous MI [32]. These studies also suggest the need for cholesterol lowering in carriers of the rs20455 allele. For example rs20455 allele carriers had a hazard ratio of 1.50 in the CARE trial [31] and odds ratio of 1.55 (95\%CI 1.14-2.09) in WOSCOPS trial [32]. For the rs20455 carriers the absolute risk reduction by pravastatin was $4.89 \%$ in the CARE trial and $5.49 \%$ in the WOSCOPS trial. In the PROSPER [33] study among the elderly population with previous CVD disease on pravastatin therapy, there was an absolute risk reduction of $6.3 \%$ in KIF6 carriers versus $1.2 \%$ in noncarriers with a $33.6 \%$ reduction of relative risk among carriers with the number needed to treat with pravastatin significantly lower in carriers compared to noncarriers (16 versus 83 ). Thus, these three trials demonstrated that KIF6 risk allele carriers had an increased risk of developing coronary events and the use of pravastatin significantly reduced the risk. Observational studies with multiple populations also found increased risk in allele carriers [34-38], Another study of 539 participants showed that the KIF6 risk variant was associated with cardiac events with a hazard ratio of 1.33 after adjustment for age, sex race, high-sensitivity C-reactive protein, and LDL-C [34]. In the ARIC $[35,36]$ study, carriers of the KIF6 719Arg risk variant had a higher incidence of $\mathrm{CHD}$ with a hazard ratio of 1.22 after model was adjusted for age and sex. In the WHS [38] study, carriers of the KIF67 719Arg risk variant had a greater CHD risk with a hazard ratio of 1.24 and a hazard ratio of 1.34 associated with risk for MI.

In contrast, other large studies have not found an association of Kif6 allele with statin response [39, 40]. The Heart Protection Study (HPS) with 18,348 randomized patients found that there was no impact of KIF6 genotype on statin therapy and vascular events across all genotypes [39, 40]. In The JUPITER Trial, Caucasian participants with low LDL-C $<130 \mathrm{mg} / \mathrm{dL}$ and elevated high-sensitivity C-reactive protein $\geq 2 \mathrm{mg} / \mathrm{L}$ were randomly assigned to rosuvastatin or placebo and followed for first major CVD event [12]. There were no differences in vascular event rates in KIF6 risk allele carriers compared to noncarriers (hazard ratio of 0.91) nor amount of LDL-C reduction. Differences in study design between the earlier statin trials and more recent trials such as HPS [39, 40] and JUPITER [12] could help explain the different findings. In the HPS placebo patients have been intensively treated with $40 \mathrm{mg}$ simvastatin for 6 weeks prior to randomization which substantially reduced LDL-C levels and according to Ference et al. [18] could attenuate risk associated with KIF6 and therefore explain the lack of a differential response to statin therapy in those with KIF6 genotype. And, similarly, in JUPITER only participants with low LDL-C levels were enrolled in the study and were treated with highdose rosuvastatin. Such contradictory findings necessitate further investigation of the effect of KIF6 polymorphism on cardiovascular health.
4.1. Limitations. Certain study limitations merit comment. First, the study was based upon a small number of subjects and the significant genetic admixture of Filipinos also presents a challenge when small population subsets are studied. The exclusion of Filipino-American men was another limitation to the study, although it should be noted that most studies include only men and women have been an understudied gender. Moreover, we have developed a successful method of recruiting women for these sorts of studies in community settings. It is not clear if this method would work for Filipino-American men.

Third, since this study was only conducted on the Filipino population in the United States, the generalizability to Filipinos residing in other countries is not known. Fourth, the use of the cross-sectional design limits examining risk factors for heart disease at a single point in time without the ability to measure cardiovascular outcomes.

\section{Conclusion}

Our results showed that $70 \%$ of a cohort of nonrelated Filipino-American women are carriers of the Trp719Arg risk allele (rs20455 SNP of the KIF6 gene). Importantly, many of these women, even those treated with statins, did not achieve guideline recommended LDL-C levels. Although larger population studies are needed to confirm our findings, the results indicate that Filipino-American women need to be more carefully monitored and treated for elevated cholesterol in order to reduce the incidence of CHD in this population.

\section{Conflict of Interests}

The authors declare that there is no conflict of interests regarding the publication of this paper.

\section{References}

[1] J. C. Cohen, "Genetic approaches to coronary heart disease," Journal of the American College of Cardiology, vol. 48, no. 9, pp. A10-A14, 2006.

[2] A. J. Lusis, A. M. Fogelman, and G. C. Fonarow, "Genetic basis of atherosclerosis: part II: clinical implications," Circulation, vol. 110, no. 14, pp. 2066-2071, 2004.

[3] G. H. Gibbons, C. C. Liew, M. O. Goodarzi et al., "Genetic markers: progress and potential for cardiovascular disease," Circulation, vol. 109, no. 25, supplement 1, pp. IV47-IV58, 2004.

[4] J. F. Peden and M. Farrall, "Thirty-five common variants for coronary artery disease: the fruits of much collaborative labour," Human Molecular Genetics, vol. 20, no. 2, pp. R198-205, 2011.

[5] The IBC 50K CAD Consortium, "Large-scale gene-centric analysis identifies novel variants for coronary artery disease," PLoS Genetics, vol. 7, no. 9, Article ID e1002260, 2011.

[6] N. Hirokawa, "Kinesin and dynein superfamily proteins and the mechanism of organelle transport," Science, vol. 279, no. 5350, pp. 519-526, 1998.

[7] H. Miki, M. Setou, K. Kaneshiro, and N. Hirokawa, "All kinesin superfamily protein, KIF, genes in mouse and human," Proceedings of the National Academy of Sciences of the United States of America, vol. 98, no. 13, pp. 7004-7011, 2001. 
[8] S. Seiler, J. Kirchner, C. Horn, A. Kallipolitou, G. Woehlke, and M. Schliwa, "Cargo binding and regulatory sites in the tail of fungal conventional kinesin," Nature Cell Biology, vol. 2, no. 6, pp. 333-338, 2000.

[9] P. Peng, J. Lian, R. S. Huang et al., "Meta-analyses of KIF6 rs20455 in coronary heart disease and statin therapeutic effect," PLoS ONE, vol. 7, no. 12, Article ID e50126, 2012.

[10] G. Wu, G. B. Li, and B. Dai, "Association of KIF6 variant with lipid level and angiographic coronary artery disease events risk in the Han Chinese population," Molecules, vol. 17, no. 9, pp. 11269-11280, 2012.

[11] D. Shiffman, D. I. Chasman, R. Y. L. Zee et al., "A kinesin family member 6 variant is associated with coronary heart disease in the Women's Health Study," Journal of the American College of Cardiology, vol. 51, no. 4, pp. 444-448, 2008.

[12] P. M. Ridker, J. G. Macfadyen, R. J. Glynn, and D. I. Chasman, "Kinesin-like protein 6 (KIF6) polymorphism and the efficacy of rosuvastatin in primary prevention," Circulation: Cardiovascular Genetics, vol. 4, pp. 312-317, 2011.

[13] D. Shiffman, M. S. Sabatine, J. Z. Louie et al., "Effect of pravastatin therapy on coronary events in carriers of the KIF6 719Arg allele from the cholesterol and recurrent events trial," The American Journal of Cardiology, vol. 105, no. 9, pp. 13001305, 2010.

[14] O. A. Iakoubova, C. H. Tong, C. M. Rowland et al., "Association of the Trp719Arg polymorphism in kinesin-like protein 6 with myocardial infarction and coronary heart disease in 2 prospective trials. The CARE and WOSCOPS trials," Journal of the American College of Cardiology, vol. 51, no. 4, pp. 435-443, 2008.

[15] Y. Li, O. A. Iakoubova, D. Shiffman, J. J. Devlin, J. S. Forrester, and H. R. Superko, "KIF6 polymorphism as a predictor of risk of coronary events and of clinical event reduction by statin therapy," The American Journal of Cardiology, vol. 106, no. 7, pp. 994-998, 2010.

[16] L. A. Bare, E. A. Ruiz-Narvaéz, C. H. Tong et al., "Investigation of KIF6 Trp719Arg in a case-control study of myocardial infarction: a Costa Rican population," PLoS ONE, vol. 5, no. 9, Article ID e13081, 2010.

[17] T. Assimes, H. Holm, S. Katherisan et al., "Lack of assoaition between the rs20455 polymorphism in Kinesin-like protein-6 and coronary artery disease in 19 case-control studies," Journal of the American College of Cardiology, vol. 56, no. 9, pp. 15521563, 2010.

[18] B. A. Ference, W. Yoo, J. M. Flack, and M. Clarke, "A common KIF6 polymorphism increases vulnerability to lowdensity lipoprotein cholesterol: two meta-analyses and a metaregression analysis," PLoS ONE, vol. 6, no. 12, Article ID e28834, 2011.

[19] L. J. Larsen, "The foreign born population in the United States: 2003," Current Population Reports, U.S. Census Bureau, Washington, DC, USA, 2004.

[20] R. A. Ursua, N. S. Islam, D. E. Aguilar et al., "Predictors of hypertension among Filipino immigrants in the Northeast US," Journal of Community Health, vol. 38, no. 5, pp. 847-855, 2013.

[21] M. R. G. Araneta, A. Grandinetti, and H. K. Chang, "A1C and diabetes diagnosis among Filipino Americans, Japanese Americans, and Native Hawaiians," Diabetes Care, vol. 33, no. 12, pp. 2626-2628, 2010.

[22] A. T. Holland, B. Zhao, E. C. Wong, S. E. Choi, N. D. Wong, and L. P. Palaniappan, "Racial/ethnic differences in control of cardiovascular risk factors among type 2 diabetes patients in an insured, ambulatory care population," Journal of Diabetes and Its Complications, vol. 27, no. 1, pp. 34-40, 2013.

[23] I. B. Ancheta, C. A. Battie, T. Tuason, and C. V. Ancheta, "A comparison of metabolic syndrome (MetS) risk factors in Filipino women and Filipino American women: A Pilot Study," Ethnicity \& Disease, vol. 22, no. 4, pp. 404-409, 2012.

[24] L. P. Palaniappan, M. R. Araneta, T. L. Assimes et al., "Call to action: cardiovascular disease in Asian Americans: a science advisory from the American Heart Association," Circulation, vol. 122, pp. 1242-1252, 2010.

[25] M. Iannone, J. Taylor, J. Chen et al., "Multiplexed single nucleotide polymorphism genotyping by oligonucleotide ligation and flow cytometry," Cytometry, vol. 39, pp. 131-140, 2000.

[26] Y. S. Kim, S. Sunwoo, H. R. Lee et al., "Determinants of non-compliance with lipid-lowering therapy in hyperlipidemic patients," Pharmacoepidemiology and Drug Safety, vol. 11, no. 7, pp. 593-600, 2002.

[27] A. M. Peterson and W. F. McGhan, "Pharmacoeconomic impact of non-compliance with statins," PharmacoEconomics, vol. 23, no. 1, pp. 13-25, 2005.

[28] K. O. Bonsu, A. Kadirvelu, and D. D. Reidpath, "Lipophilic versus hydrophilic statin therapy for heart failure: a protocol for an adjusted indirect comparison meta-analysis," Systematic Reviews, vol. 2, article 22, 2013.

[29] S. Ganesan and M. K. Ito, "Coenzyme Q10 ameliorates the reduction in GLUT4 transporter expression induced by simvastatin in 3T3-L1 adipocytes," Metabolic Syndrome and Related Disorders, vol. 11, no. 4, pp. 251-255, 2013.

[30] U. S. Food and Drug Administration, FDA Public Health Advisory for Crestor (Rosuvastatin), 2013, http://www.fda.gov/ Drugs/DrugSafety/PostmarketDrugSafetyInformationforPatientsandProviders/DrugSafetyInformationforHeathcareProfessionals/PublicHealthAdvisories/ucm051756.htm.

[31] F. M. Sacks, M. A. Pfeffer, L. A. Moye et al., "The effect of pravastatin on coronary events after myocardial infarction in patients with average cholesterol levels," The New England Journal of Medicine, vol. 335, no. 14, pp. 1001-1009, 1996.

[32] J. Shepherd, S. M. Cobbe, I. Ford et al., "Prevention of coronary heart disease with pravastatin in men with hypercholesterolemia," The New England Journal of Medicine, vol. 333, no. 20, pp. 1301-1307, 1995.

[33] J. Shepherd, G. J. Blauw, M. B. Murphy et al., "Pravastatin in elderly individuals at risk of vascular disease (PROSPER): a randomised controlled trial," The Lancet, vol. 360, no. 9346, pp. 1623-1630, 2002.

[34] M. Cushman, E. S. Cornell, P. R. Howard, E. G. Bovill, and R. P. Tracy, "Laboratory methods and quality assurance in the Cardiovascular Health Study," Clinical Chemistry, vol. 41, no. 2, pp. 264-270, 1995.

[35] L. P. Fried, N. O. Borhani, P. Enright et al., “The Cardiovascular Health Study: design and rationale," Annals of Epidemiology, vol. 1, no. 3, pp. 263-276, 1991.

[36] M. Szklo, R. Barnes, A. Folsom et al., "The Atherosclerosis Risk in Communities (ARIC) Study: design and objectives. The ARIC investigators," American Journal of Epidemiology, vol. 129, no. 4, pp. 687-702, 1989.

[37] A. D. White, A. R. Folsom, L. E. Chambless et al., "Community surveillance of coronary heart disease in the Atherosclerosis Risk in Communities (ARIC) Study: methods and initial two years' experience," Journal of Clinical Epidemiology, vol. 49, no. 2, pp. 223-233, 1996. 
[38] P. M. Ridker, N. R. Cook, I.-M. Lee et al., "A randomized trial of low-dose aspirin in the primary prevention of cardiovascular disease in women," The New England Journal of Medicine, vol. 352, no. 13, pp. 1293-1304, 2005.

[39] J. C. Hopewell, S. Parish, R. Clarke et al., "No impact of KIF6 Genotype on vascular risk and statin response among 18,348 randomized patients in the heart protection study," Journal of the American College of Cardiology, vol. 57, no. 20, pp. 20002007, 2011.

[40] J. C. Hopewell, S. Parish, A. Offer et al., "Impact of common genetic variation on response to simvastatin therapy among 18 705 participants in the Heart Protection Study," European Heart Journal, vol. 34, pp. 982-992, 2013. 


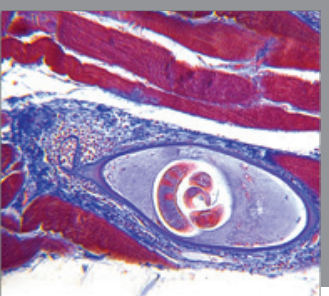

Gastroenterology

Research and Practice
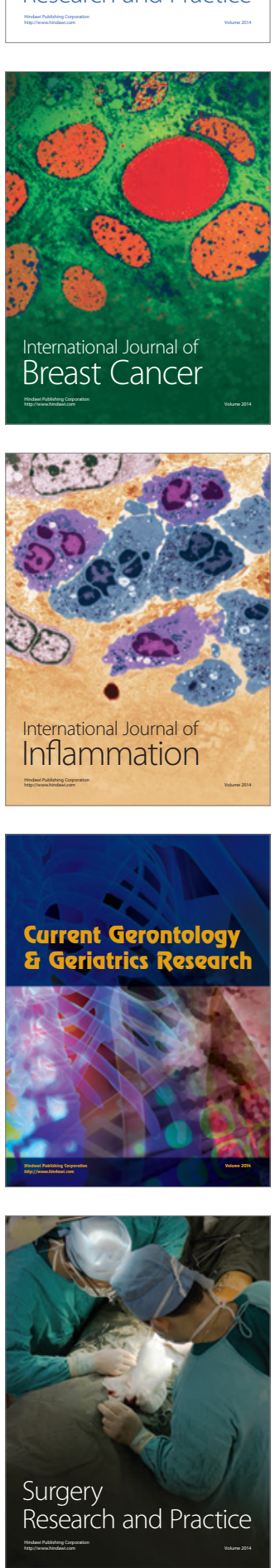

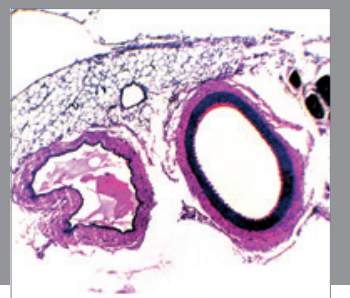

International Journal of Hypertension
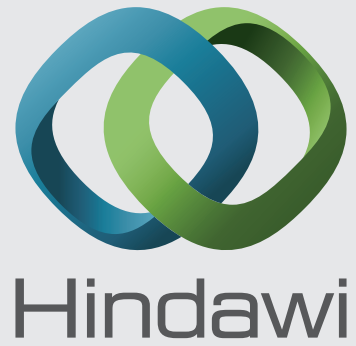

Submit your manuscripts at http://www.hindawi.com
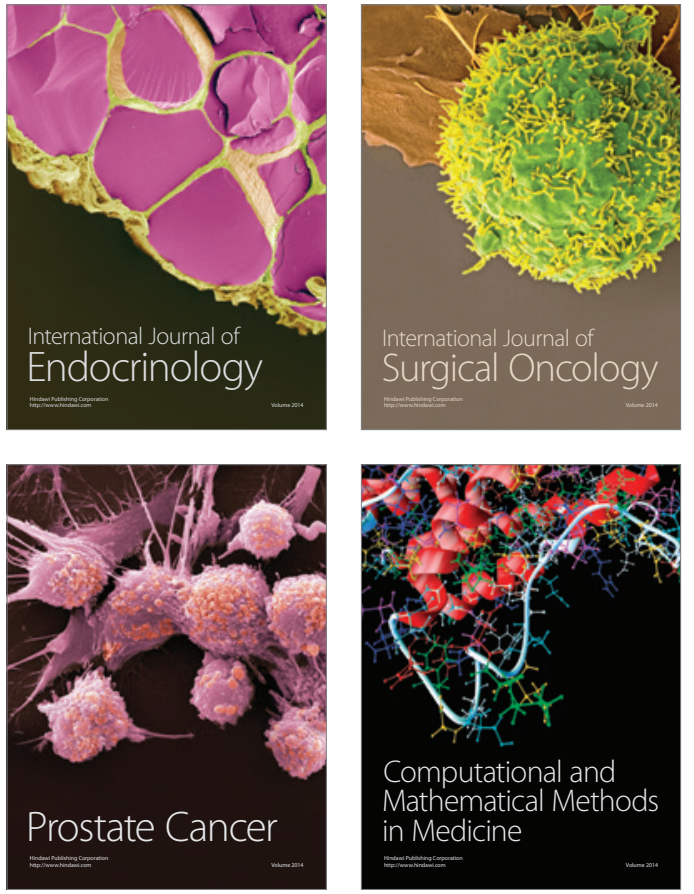
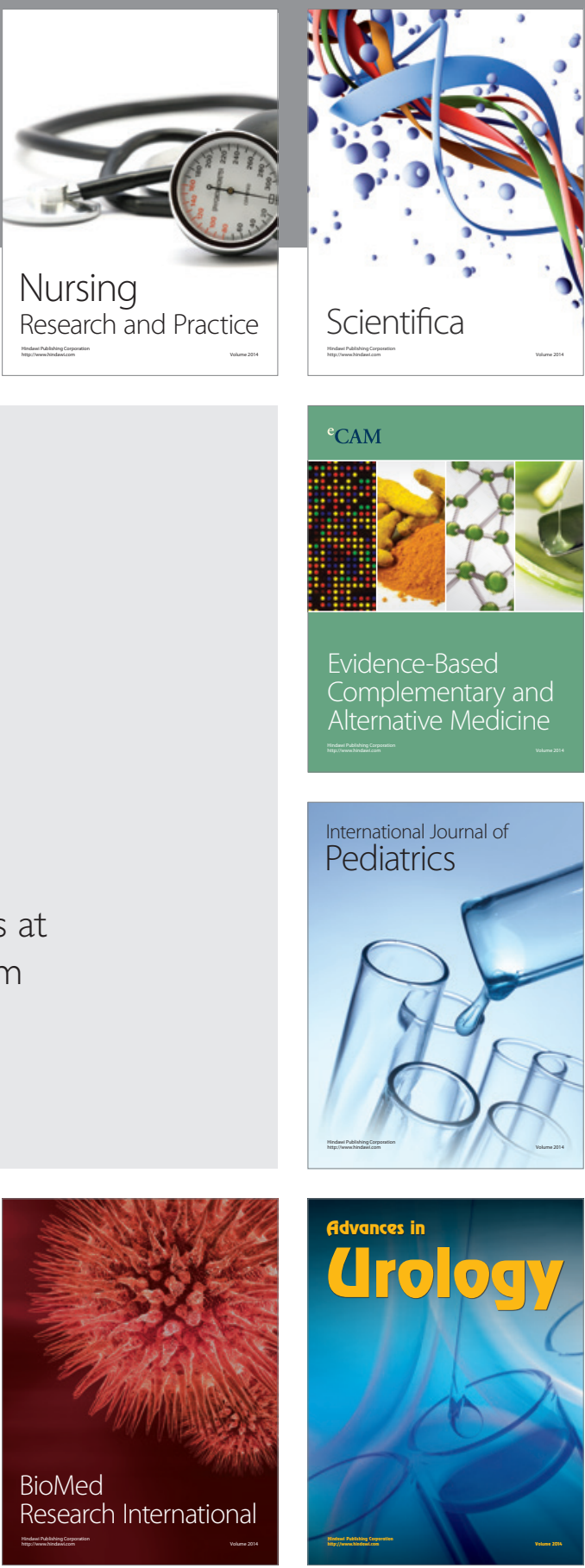

Nursing

Research and Practice

Scientifica

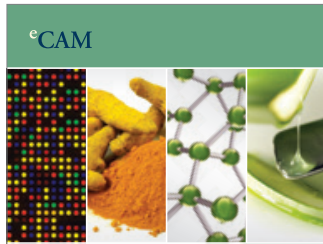

Evidence-Based

Complementary and Alternative Medicine
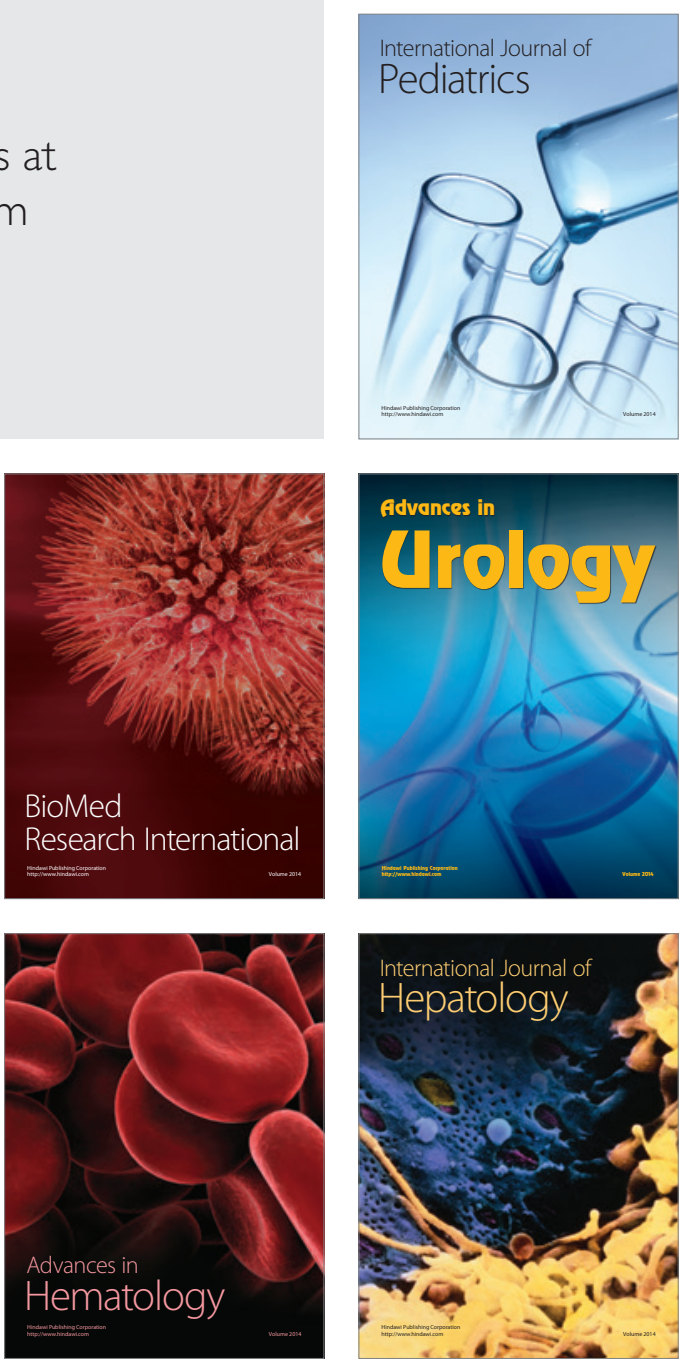